\title{
Loss of peristalsis of the esophagus due to diffuse esophageal leiomyomatosis
}

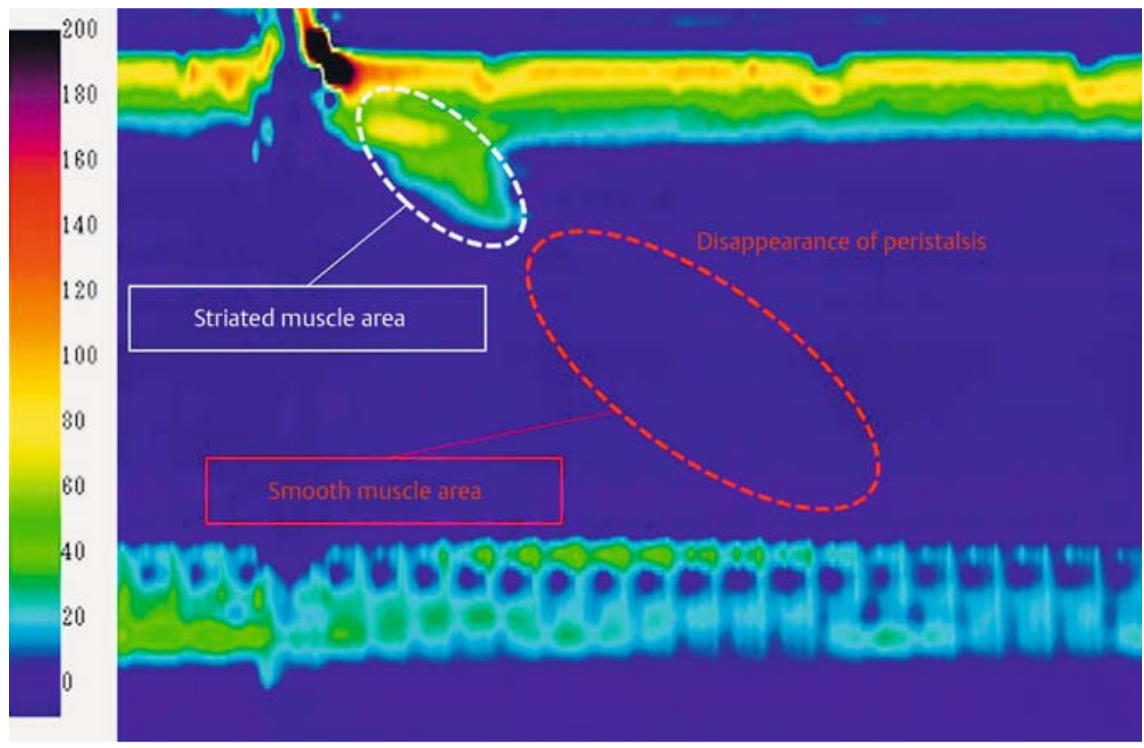

- Fig. 1 High resolution manometry findings showing the disappearance of peristalsis of the smooth muscle area, whereas peristalsis of the striated muscle area was preserved.
A 15-year-old girl was referred to our hospital with a tumor in the mediastinum that had been found on a chest radiograph during a medical check-up. An esophagram showed a large mediastinal mass (60 $\mathrm{mm}$ in diameter) ( $\triangleright$ Video 1 ). No peristalsis of the thoracic esophagus (smooth muscle area) was apparent, and dilatation of the esophagus and retention of the barium were observed on the proximal side of the tumor. High resolution manometry (HRM) findings showed complete loss of peristalsis of the smooth muscle area, whereas peristalsis of the striated muscle area was preserved ( $\triangleright$ Fig. 1). Although the esophageal lumen was narrowed by the tumor, the endoscope could be passed through it ( Fig. 2). Computed tomography (CT) scanning demonstrated a mildly enhancing tumor, which was circumferentially surrounding the lower esophagus ( Fig.3). On T1-weighted magnetic resonance imaging (MRI), the tumor had a relatively high signal, which was the same as that of other muscular organs ( Fig.4).
The patient underwent endoscopic ultrasound-guided fine needle aspiration (EUS-FNA), with the endoscopic ultrasound showing that the tumor had a mosaic echo, and that there was a clear boundary between the tumor and other organs ( $\triangleright$ Fig.5). Biopsy specimens

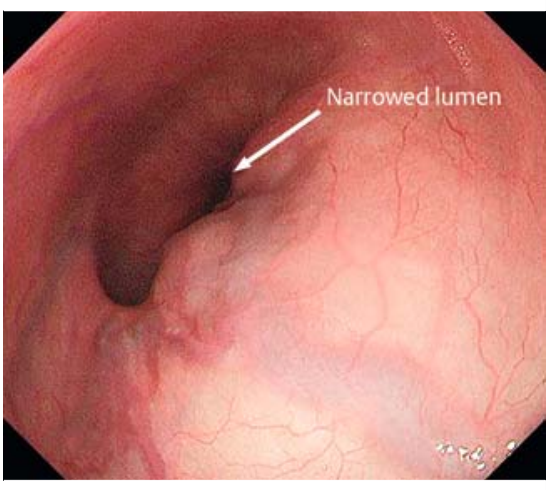

- Fig. 2 Endoscopic image showing the narrowed esophageal lumen caused by a huge submucosal tumor.

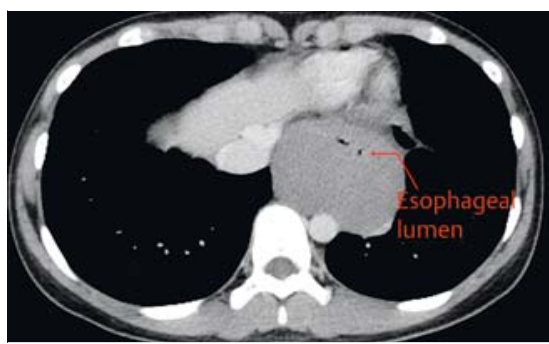

- Fig. 3 Computed tomography (CT) scan showing a mildly enhancing tumor with a diameter of $60 \mathrm{~mm}$, which had circumferentially surrounded the lower esophagus. The tumor was not invading other organs.

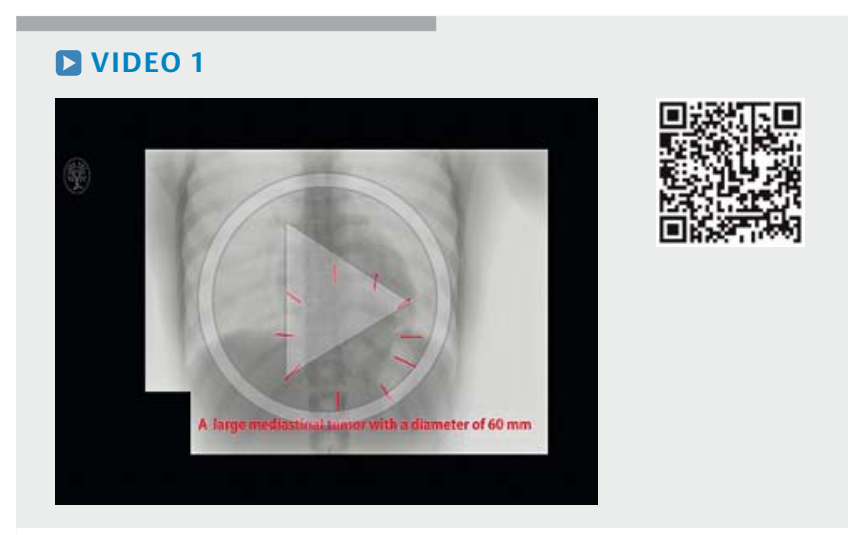

- Video 1: Results of the esophagram showing a large mediastinal tumor with a diameter of $60 \mathrm{~mm}$. The esophageal lumen is narrowed by the tumor, and there is no peristalsis of the smooth muscle area visible. Dilatation of the esophagus and retention of the barium are observed on the proximal side of the tumor. 


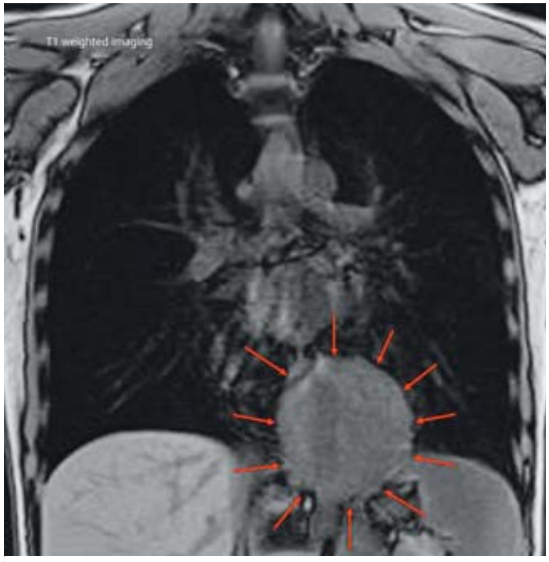

- Fig. 4 The relatively high signal of the tumor was similar to that of other muscular organs on the T1-weighted magnetic resonance imaging (MRI) scan, indicating that the tumor tissue had similar properties to that of muscle.

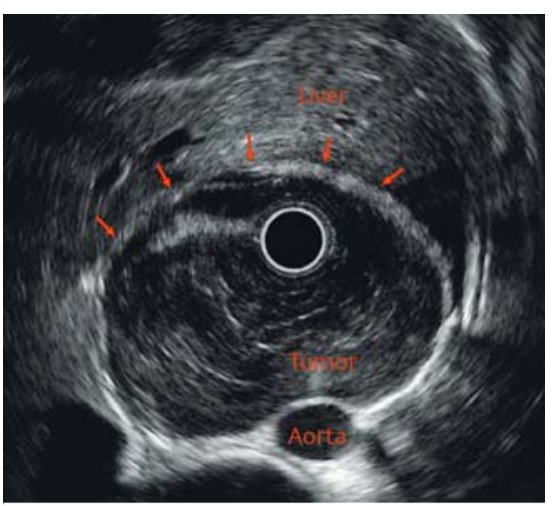

- Fig. 5 Endoscopic ultrasound image showing that the tumor had a mosaic echo and that there was a clear boundary between the tumor and other organs.

showed spindle cells without atypia on hematoxylin and eosin (H\&E) staining; they were positive for smooth muscle actin and desmin, and negative for c-kit by immunohistochemistry (> Fig.6). A diagnosis of diffuse esophageal leiomyomatosis (DEL) was made but, as she was asymptomatic, surgery was not indicated.

DEL is a rare, benign disease characterized by diffuse hypertrophy of the esophageal muscular layer; some cases of DEL are associated with Alport syndrome [1 -4]. Although patients with DEL usually present with dysphagia [1,2], our patient was asymptomatic with no signs of Alport syndrome. Esophageal motility in DEL investigated by HRM has rarely been reported [5]. This is the first report

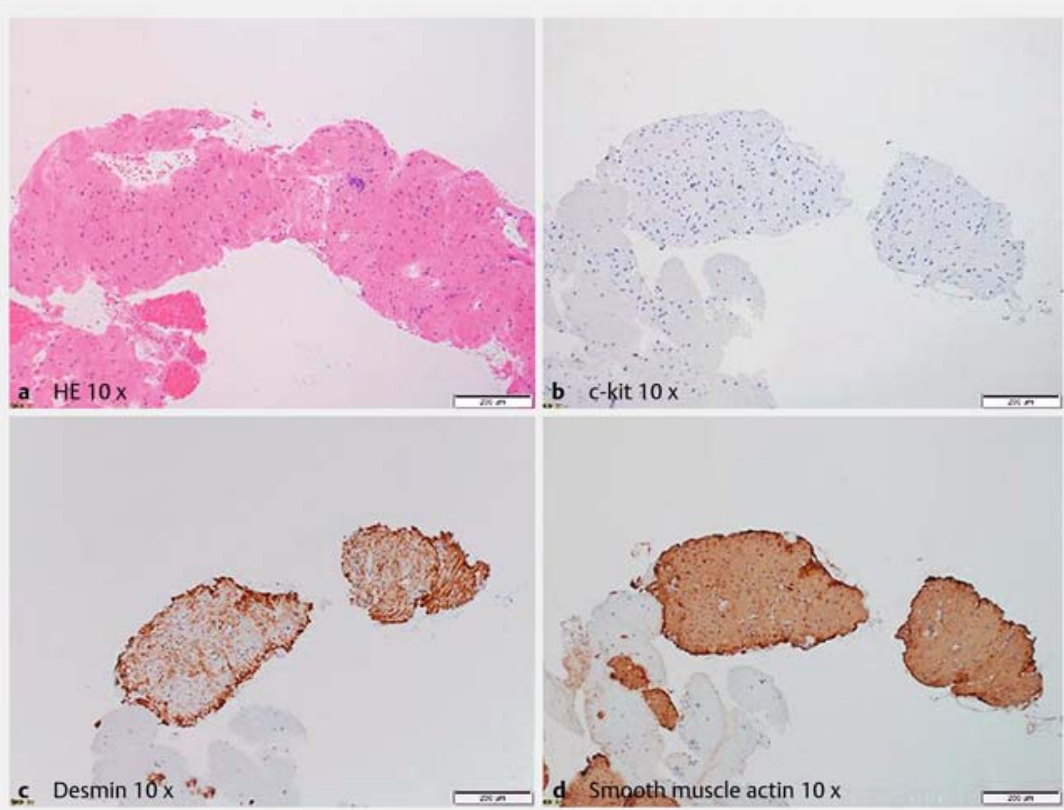

- Fig. 6 Spindle cells without atypia are seen using hematoxylin and eosin (H\&E) staining. Immunohistochemical staining shows the cells are positive for smooth muscle actin and desmin, but negative for c-kit, indicating the histopathological diagnosis of leiomyoma.

showing loss of peristalsis of the esophagus due to DEL, although the mechanism of this remains unknown.

Endoscopy_UCTN_Code_CCL_1AB_2AC_3AH

\section{Competing interests}

None

The Authors

Kazuya Takahashi, Yui Ishii, Kazunao Hayashi, Satoshi Ikarashi, Hirokazu Kawai, Yuichi Sato, Shuji Terai

Division of Gastroenterology and Hepatology, Graduate School of Medical and Dental Science, Niigata University, Niigata, Japan

\section{Corresponding author}

\section{Kazuya Takahashi, MD, PhD}

Division of Gastroenterology and

Hepatology, Graduate School of Medical and Dental Science, Niigata University, 1-757

Asahimachi-dori, Chuo-ku, Niigata City,

Niigata, 951-8510, Japan

Fax: +81-25-2270776

kazuya911@med.niigata-u.ac.jp

\section{References}

[1] Crouch G, Devitt PG, Thompson SK. Diffuse esophageal leiomyomatosis. Dis Esophagus 2011; 24: 145 - 146

[2] Pujol ], Parés D, Mora L et al. Diagnosis and management of diffuse leiomyomatosis of the oesophagus. Dis Esophagus 2000; 13: $169-171$

[3] Uliana V, Marcocci E, Mucciolo M et al. Alport syndrome and leiomyomatosis: The first deletion extending beyond COL4A6 intron 2. Pediatr Nephrol 2011; 26: 717 724

[4] Liu W, Wong JKL, He Q et al. Chinese family with diffuse oesophageal leiomyomatosis: a new COL4A5/COL4A6 deletion and a case of gonosomal mosaicism. BMC Med Genet 2015; 16: 49

[5] Katzka DA, Smyrk TC, Chial H] et al. Esophageal leiomyomatosis presenting as achalasia diagnosed by high-resolution manometry and endoscopic core biopsy. Gastrointest Endosc 2012; 76: 216-217

\section{Bibliography}

DOI http://dx.doi.org/10.1055/s-0043-100691 Endoscopy 2017; 49: E95-E96

(c) Georg Thieme Verlag KG

Stuttgart · New York

ISSN 0013-726X 